\title{
Solution of the Nonlinear High-Fidelity Generalized Method of Cells Micromechanics Relations via Order-Reduction Techniques
}

\author{
Trenton M. Ricks $\mathbb{D}^{1,2}$ Thomas E. Lacy Jr., ${ }^{1}$ Brett A. Bednarcyk, ${ }^{2}$ \\ Annika Robens-Radermacher, ${ }^{3}$ Evan J. Pineda, ${ }^{2}$ and Steven M. Arnold ${ }^{2}$ \\ ${ }^{1}$ Department of Aerospace Engineering, Mississippi State University, P.O. Box A, Mississippi State, MS 39762, USA \\ ${ }^{2}$ Multiscale and Multiphysics Modeling Branch, Materials and Structures Division, NASA Glenn Research Center, \\ 21000 Brookpark Rd., MS 49-7, Cleveland, OH 44135, USA \\ ${ }^{3}$ BAM Federal Institute for Material Research and Testing, Unter den Eichen 87, 12205 Berlin, Germany
}

Correspondence should be addressed to Trenton M. Ricks; trenton.m.ricks@nasa.gov

Received 17 November 2017; Accepted 29 January 2018; Published 28 February 2018

Academic Editor: Jose Merodio

\begin{abstract}
Copyright (C) 2018 Trenton M. Ricks et al. This is an open access article distributed under the Creative Commons Attribution License, which permits unrestricted use, distribution, and reproduction in any medium, provided the original work is properly cited.

The High-Fidelity Generalized Method of Cells (HFGMC) is one technique, distinct from traditional finite-element approaches, for accurately simulating nonlinear composite material behavior. In this work, the HFGMC global system of equations for doubly periodic repeating unit cells with nonlinear constituents has been reduced in size through the novel application of a Petrov-Galerkin Proper Orthogonal Decomposition order-reduction scheme in order to improve its computational efficiency. Order-reduced models of an E-glass/Nylon 12 composite led to a 4.8-6.3x speedup in the equation assembly/solution runtime while maintaining model accuracy. This corresponded to a $21-38 \%$ reduction in total runtime. The significant difference in assembly/solution and total runtimes was attributed to the evaluation of integration point inelastic field quantities; this step was identical between the unreduced and order-reduced models. Nonetheless, order-reduced techniques offer the potential to significantly improve the computational efficiency of multiscale calculations.
\end{abstract}

\section{Introduction}

The High-Fidelity Generalized Method of Cells (HFGMC) is a micromechanics technique that can be used to simulate nonlinear composite materials [1]. The core computational effort of this method involves repeatedly finding the solution to sets of simultaneous linear algebraic equations in order to determine local/global field quantities and effective properties for heterogeneous materials with a periodic microstructure. However, when material nonlinearity is admitted, the computational runtimes can become excessive, particularly as the problem size is increased due to a more detailed microstructural representation. Nonlinear analyses of such detailed, high-fidelity repeating unit cells (RUCs) are needed to accurately simulate realistic composite microstructures necessary for process modeling, prediction of residual stress states, progressive failure analysis, and other computational predictions that depend heavily on subscale features. The use of order-reduction techniques is one possibility of improving the computational efficiency of high-fidelity analyses. Furthermore, although HFGMC is fundamentally distinct and more computationally efficient than traditional finiteelement (FE) approaches [1], both methods are relatively inefficient for multiscale simulations of realistic composite microstructures.

Proper Orthogonal Decomposition (POD) $[2,3]$ and Proper Generalized Decomposition (PGD) $[4,5]$ are two commonly used order-reduction approaches. In order to generate an order-reduced model using POD, the full solution to a particular problem (often found by solving a set of simultaneous equations) must be known a priori. If this solution cannot be practically obtained due to model size or 
computational limits, PGD can be used to generate an orderreduced model. However, for most solid mechanics problems of interest, a priori solutions can be easily obtained. More detailed information on PGD can be found in the review article by Chinesta et al. [4]. In this study, a POD approach was used due to its wide use in the literature and ease of implementation.

A significant number of FE studies have employed POD to generate order-reduced models that reduce the dimensionality of the ensuing large set of simultaneous equations. The goal of POD is to generate a set of basis vectors capable of capturing the dominant components of a system, optimally represent a full set of equations, and provide a mapping relationship between the unreduced and orderreduced domains. In this context, an order-reduced POD approach has two main components: (i) approximation of the solution to a set of equations and (ii) projection to the order-reduced domain. In general, FE-based POD techniques employ Galerkin projection (i.e., the projection is performed with the same set of basis vectors used for approximation). Carlberg et al. [6] noted that Galerkin projection may not be optimal in the presence of nonlinearity and can lead to computational instabilities. A more complex Petrov-Galerkin POD method was developed to overcome these limitations by modifying the form of the projection at the cost of some added calculations [6].

While POD-based order-reduction techniques have been commonly used to solve problems in computational fluid dynamics [7-9], these techniques have also been extended to include nonlinear solid mechanics problems [6, 10-14]. For instance, Radermacher et al. [10] were able to demonstrate improvements of the computational speed by a factor of 60-260 by employing a POD-based order-reduction technique in the analysis of an inelastic metal matrix composite. POD techniques have also been implemented within a multiscale framework. Multiscale methods are often based on an $\mathrm{FE}^{2}[15,16]$ modeling approach, wherein a microscale FE model is called at each integration point within a macroscale FE model. Yvonnet and He [13] were able to achieve significant computational and memory savings for multiscale simulations of hyperelastic media. Radermacher et al. [10] demonstrated two orders of magnitude speedup in the computational time of nonlinear multiscale simulations by implementing POD at the microscale. Similarly, Ricks et al. [17] obtained significant computational savings by imbedding HFGMC within a macroscale linearly elastic FE model.

Several authors have also proposed methods to modify/update the original set of basis vectors in order to achieve better computational performance. Hernández et al. [12] formed a set of basis vectors by accounting for all elastic modes and only the essential inelastic modes. Ryckelynck [18] developed a procedure to adaptively update the subspace spanned by the original set of basis vectors during an analysis. Additional computational savings were achieved by using only a subset of the FEs to control the adaptive process [18]. This "hyperreduction" approach is similar in concept to the discrete empirical interpolation method [19] and gappy POD technique [20, 21]. Kerfriden et al. [14] proposed updating the original set of basis vectors using appropriately normalized unconverged/converged iterative solutions.

In the present work, the HFGMC global system of equations for doubly periodic RUCs with nonlinear constituents is reduced in size through the use of POD. This approach was previously shown to yield significant computational savings when applied to the HFGMC equations for linearly elastic materials only [17]. The order-reduced HFGMC models are then compared to the traditional HFGMC approach for multiple RUC discretizations in order to assess their accuracy and computational efficiency.

\section{High-Fidelity Generalized Method of Cells (HFGMC)}

The HFGMC is a micromechanics technique used for modeling heterogeneous materials [1] and is an adaptation of classical homogenization theory [22-24]. The HFGMC has been extensively validated and shown to accurately calculate both effective properties and thermoinelastic material behavior for a wide range of composite material systems (see [1] for a partial summary). In contrast to the generalized method of cells [1], the HFGMC gives a higher accuracy in the subcell stress/strain fields, at an increased computational cost, by employing a higher-order subcell displacement field. Using the HFGMC, a doubly periodic RUC is discretized into an arbitrary number of subcells (see Figure 1). A doubly periodic RUC may be defined in the $y_{2}-y_{3}$ plane and is discretized into $N_{\beta}$ and $N_{\gamma}$ subcells along the $y_{2}$-direction (height) and the $y_{3}$-direction (width), respectively, while any inhomogeneities/inclusions (e.g., fibers) extend infinitely in the $y_{1}$-direction (length). A local $\bar{y}_{2}{ }^{(\beta)}-\bar{y}_{3}{ }^{(\gamma)}$ coordinate system may be defined relatively to the centroid of each subcell. The height and length of each subcell are given by $h_{\beta}$ and $l_{\gamma}$, respectively. The discussion that follows presents key aspects of the HFGMC formulation that are relevant to this study. An exhaustive derivation of the HFGMC can be found in [1].

2.1. HFGMC Subcell Equations. Each subcell in an RUC is assigned material properties and a constitutive law to describe the local material behavior. The constitutive law for thermoinelastic materials is given by

$$
\boldsymbol{\sigma}^{(\beta \gamma)}=\mathbf{C}^{(\beta \gamma)}\left(\boldsymbol{\varepsilon}^{(\beta \gamma)}-\boldsymbol{\varepsilon}^{T^{(\beta \gamma)}}-\boldsymbol{\varepsilon}^{I^{(\beta \gamma)}}\right),
$$

where $\boldsymbol{\sigma}^{(\beta \gamma)}, \mathbf{C}^{(\beta \gamma)}, \boldsymbol{\varepsilon}^{(\beta \gamma)}, \boldsymbol{\varepsilon}^{T^{(\beta \gamma)}}$, and $\boldsymbol{\varepsilon}^{I^{(\beta \gamma)}}$ are the stress, elastic stiffness, total strain, thermal strain, and inelastic strain tensors, respectively. The stress tensor is used to calculate surface-averaged tractions, $\mathbf{t}^{(\beta \gamma)}$, along the edges of a subcell as a function of the unknown fluctuating displacements. The computational efficiency of the HFGMC can be significantly improved by reformulating $\mathbf{t}^{(\beta \gamma)}$ to be a function of surfaceaveraged fluctuating displacements (unknowns) [25, 26]. A linear system of 12 equations can be derived and expressed as

$$
\mathbf{t}^{(\beta \gamma)}=\mathbf{K}^{(\beta \gamma)} \overline{\mathbf{u}}^{(\beta \gamma)}+\mathbf{f}^{(\beta \gamma)}+\mathbf{g}^{(\beta \gamma)},
$$




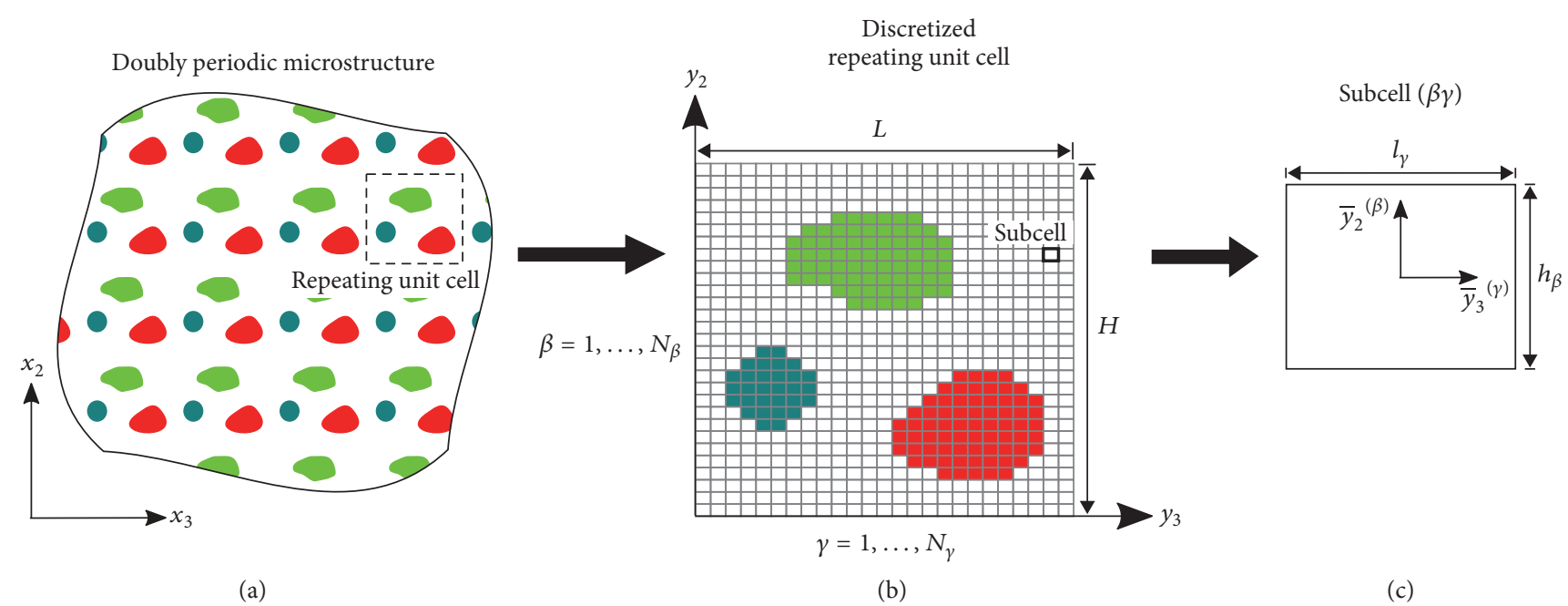

FIGURE 1: A heterogeneous composite with a doubly periodic microstructure comprised of (a) multiple RUCs. (b) A single RUC of dimensions $H \times L$ comprised of a number of individual subcells. (c) An individual subcell of dimensions $h_{\beta} \times l_{\gamma}$. Here, $x_{i}, y_{i}$, and $\overline{y_{i}}$ refer to global, RUC, and subcell coordinates, respectively $(i=1, \ldots, 3)$.

where $\overline{\mathbf{u}}^{(\beta \gamma)}$ represents the unknown surface-averaged fluctuating displacements, $\mathbf{f}^{(\beta \gamma)}$ is a vector containing subcell material properties and macroscale strain components, and $\mathbf{g}^{(\beta \gamma)}$ is a vector containing thermoinelastic traction components. The $12 \times 12$ subcell stiffness matrix, $\mathbf{K}^{(\beta \gamma)}$, contains subcell material properties and dimensions and does not depend on any inelastic quantities.

2.2. HFGMC Global Equations. By imposing interfacial traction and displacement continuity conditions on the interior subcell boundaries and periodic boundary conditions on the RUC boundaries, an assembled, linear system of equations can be derived [1]. For perfectly bonded constituents, the reformulated HFGMC relationships for a given RUC can be expressed as a square system of $n=6 N_{\beta} N_{\gamma}$ equations of the form

$$
\mathbf{K U}=\mathbf{f}+\mathbf{g},
$$

where $\mathbf{K}$ is a sparse, unsymmetrical matrix that is a function of elastic properties and geometries of the subcells, the vector $\mathbf{U}$ represents the unknown surface-averaged fluctuating displacements for each subcell, $\mathbf{f}$ is a vector containing the material properties of the subcells and the applied RUC average strains, and $\mathbf{g}$ is a vector containing material properties/dimensions and the thermoinelastic tractions that are dependent on $\mathbf{U}$. Unlike traditional nonlinear FE approaches [27], $\mathbf{K}$ does not change iteratively (i.e., it does not depend on $\mathbf{U}$ ) in the presence of material nonlinearity. All nonlinear contributions to (3) are accounted for in g. In the presence of inelasticity, these equations must be iteratively solved at each loading increment, and the solution is used to determine subcell stresses and strains. In the present HFGMC formulation, each row of $\mathbf{K}$ effectively represents a traction continuity equation between two subcells. The terms containing the unknown surface-averaged fluctuating displacements are collected on the left-hand side of (3), and all other terms are collected on the right-hand side. In general, $\mathbf{K}$ must be assembled each time the constituent properties of a subcell change.

2.3. HFGMC Solution Procedure. In a typical HFGMC analysis, the assembled HFGMC system of $n$ equations for an RUC is assembled multiple times in order to determine the mechanical strain concentration tensor for a given periodic microstructure and to solve for local and global (homogenized) field quantities for a given loading. In general, the equations are assembled and solved six times (independently varying each strain component) to establish the mechanical strain concentration tensor [1]. This tensor is used to calculate the effective elastic stiffness and thermal stress tensors of the composite. The mechanical strain concentration tensor does not depend on the inelastic material state [1]. If all elastic material properties are temperature independent, this step is only performed for the first loading increment (i.e., the effective properties are constant). When thermoelastic/thermoinelastic materials are considered, this step must be performed every loading increment where a temperature change occurs.

Additionally, for each load increment, an iterative solution procedure is required to achieve converged inelastic fields. The Mendelson method [28, 29] was used to integrate the classical plasticity equations at user-specified integration points within a subcell. In the HFGMC, the global RUC system of equations is solved two times per iteration per increment: once to solve the actual boundary value problem under consideration and another to update the inelastic field quantities for the next iteration/increment. Order-reduction techniques are hence an attractive option to reduce the computational cost associated with repeatedly assembling/solving the HFGMC system of equations. 


\section{Order-Reduction Concepts Applied to the HFGMC}

3.1. Proper Orthogonal Decomposition (POD). As previously mentioned, POD is a technique that can be used to efficiently represent a large system of equations. Suppose that the solution to (3) (i.e., $\mathrm{U}$ of length $n$ ) can be obtained a priori. The vector $\mathbf{U}$ can be expressed by $\mathbf{U}=\mathbf{V w}$, where $\mathbf{V}=$ $\left[\mathbf{V}_{\mathbf{1}}, \mathbf{V}_{2}, \ldots, \mathbf{V}_{\mathbf{n}}\right]$ is a set of $n$ arbitrary orthonormal basis vectors that span the solution space and $\mathbf{w}$ is a coefficient vector of length $n$. The goal of POD is to determine an approximate solution to $\mathbf{U}, \widehat{\mathbf{U}}=\widehat{\mathbf{V}} \widehat{\mathbf{w}}$, where $\widehat{\mathbf{V}}=\left[\mathbf{V}_{1}, \mathbf{V}_{2}, \ldots, \mathbf{V}_{\mathbf{k}}\right]$ is a set of the first $k$ vectors of $\mathbf{V}, \widehat{\mathbf{w}}$ is a vector comprised of the first $k$ components of $\mathbf{w}$, and $k \leq n$. Note that, for optimal computational performance, $k \ll n$.

The method of snapshots [30] was used to determine the set of $k$ orthonormal basis vectors and, hence, the size of the reduced set of equations. Suppose that the solution to (3) (i.e., U) for an RUC under a particular set of applied strains/stresses is known at a given loading increment (or iteration). This solution (i.e., a "snapshot") can be assigned to the first column of a new matrix, M. Additional columns of $\mathbf{M}$ can be populated using any converged (or preconverged) incremental solution to (3) for a given RUC architecture. A singular value decomposition (SVD) of the snapshot matrix, $\mathbf{M}$, can be performed and is expressed as

$$
\mathbf{M}=\mathbf{V} \Sigma \mathbf{Z}^{T},
$$

where $\mathbf{V}$ and $\mathbf{Z}$ are the left- and right-singular vectors, respectively, and $\Sigma$ is a diagonal matrix of singular values arranged in descending order. The matrix $\mathbf{V}$ is then used to populate $\widehat{\mathbf{V}}[2]$.

In this study, POD is applied to solid mechanics problems involving generalized Hooke's law including plasticity, and the snapshot matrix is assembled using individual solutions of (3) (i.e., fluctuating displacements within the RUC). POD is used to generate the best statistical fit to this multidimensional data set but is completely independent of the physical nature and origin of the data [12]. Hence, POD has been successfully applied to wide variety of physics-based (e.g., fluid dynamics [7-9]) and non-physics-based (e.g., image recognition [20]) problems.

3.2. Order-Reduced HFGMC. Consider the HFGMC system of equations given by (3) (referred to herein as the reference solution). As a result of performing POD, an approximate solution for $\mathbf{U}$ can be expressed by $\widehat{\mathbf{U}}=\widehat{\mathbf{V}} \widehat{\mathbf{w}}$ where $\widehat{\mathbf{w}}$ can be referred to as the order-reduced solution vector. This approximate solution is substituted into (3) and results in an overdetermined system of linear equations ( $n$ equations with $k$ unknowns, $k<n$ ) and a residual, $\mathbf{r}$.

$$
\mathbf{K} \widehat{\mathbf{V}} \widehat{\mathbf{W}}=\mathbf{f}+\mathbf{g}+\mathbf{r} .
$$

The residual effectively contains contributions that fall outside of the subspace spanned by $\widehat{\mathbf{V}}$. This implies that $\widehat{\mathbf{V}}^{\mathrm{T}} \mathbf{r}=$ $\mathbf{0}$ since each basis vector in $\widehat{\mathbf{V}}$ is orthogonal to $\mathbf{r}$ (i.e., the contribution from the remaining basis vectors in $\mathbf{V}$ ). The residual can be eliminated from (5) by multiplying each side by $\widehat{\mathbf{V}}^{\mathbf{T}}$. This imposes the orthogonality constraints on the residual and results in a reduced set of $k \times k$ equations.

$$
\widehat{\mathbf{V}}^{\mathrm{T}} \mathbf{K} \widehat{\mathbf{V}} \widehat{\mathbf{w}}=\widehat{\mathbf{V}}^{\mathrm{T}}(\mathbf{f}+\mathbf{g})
$$

In effect, the same basis vectors are used for both approximating the reference solution and performing the projection to the reduced system. This is commonly referred to as Galerkin-based POD (cf. [6]). However, for nonlinear HFGMC problems, this Galerkin POD approach led to numerical instabilities. Petrov-Galerkin projection was used to overcome these instabilities [6]. Rather than performing the projection by multiplying (5) by $\widehat{\mathbf{V}}^{\mathrm{T}}$, it can be multiplied by $\widehat{\mathbf{V}}^{\mathrm{T}} \mathbf{K}^{\mathrm{T}}$ resulting in a reduced set of $k \times k$ equations; that is,

$$
\widehat{\mathbf{V}}^{\mathrm{T}} \mathbf{K}^{\mathrm{T}} \mathbf{K} \widehat{\mathbf{V}} \widehat{\mathbf{w}}=\widehat{\mathbf{V}}^{\mathrm{T}} \mathbf{K}^{\mathrm{T}}(\mathbf{f}+\mathbf{g})
$$

It should be noted that in order to set up the reduced set of equations, the $n \times n$ matrix $\mathbf{K}$ and $n \times 1$ vectors $\mathbf{f}$ and $\mathbf{g}$ must be determined. The $k \times k$ reduced stiffness matrix, $\widehat{\mathbf{K}}=\widehat{\mathbf{V}}^{\mathrm{T}} \mathbf{K}^{\mathrm{T}} \mathbf{K} \widehat{\mathbf{V}}$, will only change if subcell properties are updated (e.g., due to a temperature change, damage, etc.). The approximate reference solution can be recovered by using the relationship $\widehat{\mathbf{U}}=\widehat{\mathbf{V}} \widehat{\mathbf{w}}$ once (7) is solved. In effect, the original set of $n=6 N_{\beta} N_{\gamma}$ equations can be converted into a potentially much smaller set of $k$ equations and solved. However, by only including $k$ of the $n$ orthonormal basis vectors, an error is introduced. The goal of an order-reduction technique in this context is to determine the smallest system of equations while minimizing the approximation error. An accurate orderreduced model can likely be generated provided that the orthonormal basis vectors capture the variation in input parameters (e.g., material properties, loading conditions). It is important to note that the generation of the orderreduced model is performed as part of an independent ("offline") step using solutions obtained from running one or multiple predetermined representative problems of interest. Hence, while this one-time step does require some added computational time, the benefit of order-reduced approaches can be more readily observed when a particular problem is repeatedly solved (e.g., Monte Carlo, optimization, or multiscale techniques).

\section{Microscale Simulations of Thermoinelastic Composites}

4.1. Analysis Details. The computational efficiency of the order-reduced nonlinear HFGMC method was evaluated for an E-glass fiber and Nylon 12 matrix composite system. The E-glass fiber was assumed to be isotropic and linearly elastic and was assigned temperature-independent material properties [31]. The Nylon 12 matrix was assumed to be isotropic with an elastic-perfectly plastic material response and temperature-dependent material properties [32, 33]. The applicable Young's modulus $(E)$, Poisson's ratio $(\nu)$, secant coefficient of thermal expansion (CTE), and yield stress $\left(\sigma_{y}\right)$ are presented in Table 1 as a function of temperature $(T)$. 
TABLE 1: Fiber and matrix constituent properties.

\begin{tabular}{lccccc}
\hline & $T\left({ }^{\circ} \mathrm{C}\right)$ & $E(\mathrm{MPa})$ & $\nu$ & $\sigma_{y}(\mathrm{MPa})$ & - \\
\hline Fiber & - & 74000 & 0.20 & 54.0 & $\mathrm{CTE}\left(\mu \varepsilon /{ }^{\circ} \mathrm{C}\right)$ \\
\hline \multirow{4}{*}{ Matrix } & -25 & 2100 & 0.36 & 43.9 & 158.0 \\
& 0 & 1400 & 0.36 & 28.0 & 158.0 \\
& 23 & 950 & 0.36 & 18.0 & 158.0 \\
\hline
\end{tabular}

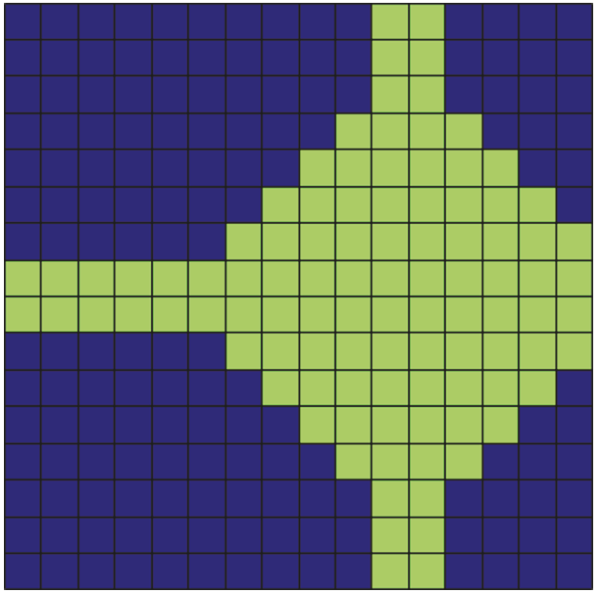

(a)

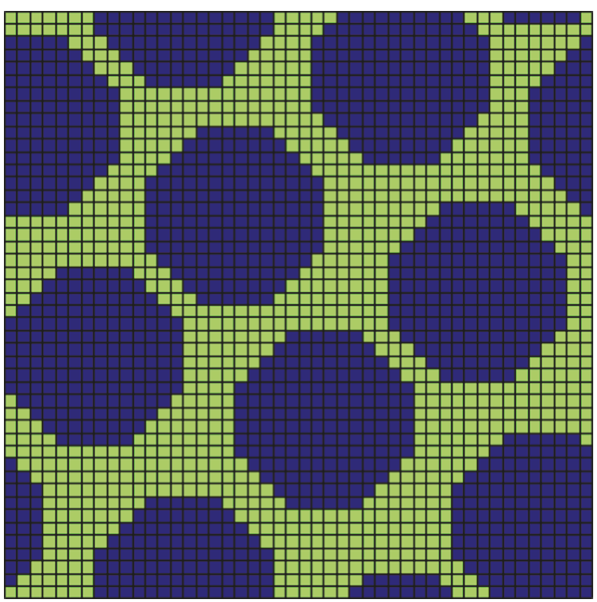

(c)

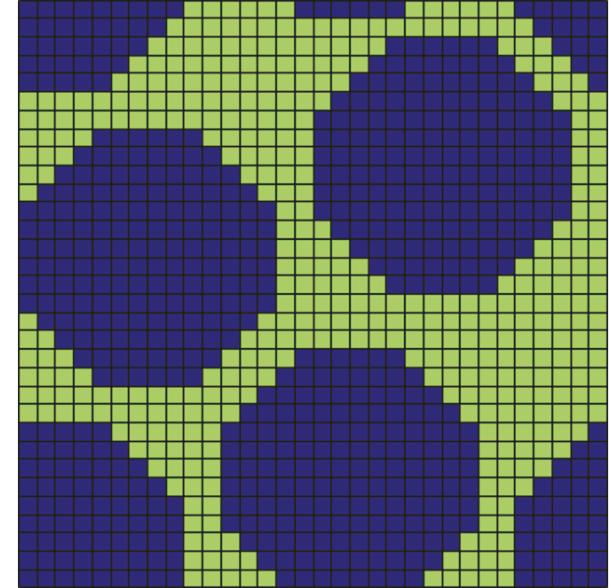

(b)

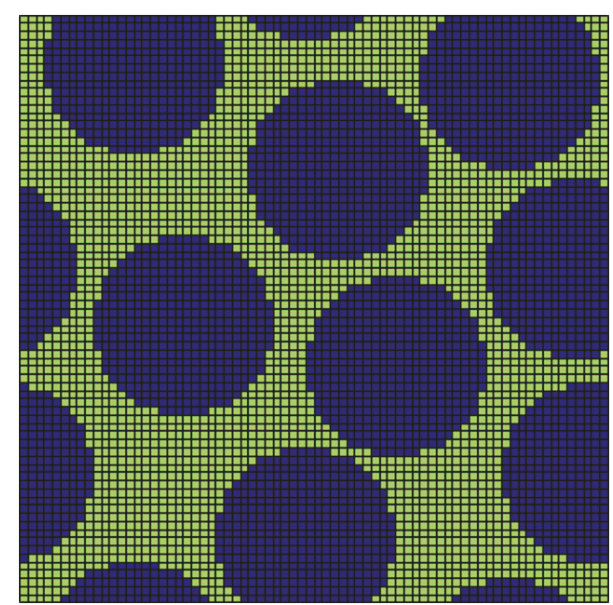

(d)

FIGURE 2: Four randomized RUC architectures comprised of (a) 256, (b) 1024, (c) 2116, and (d) 5184 subcells where blue indicates an E-glass fiber subcell and green indicates a Nylon 12 matrix subcell.

Ricks et al. [17] previously demonstrated that the computational efficiency of the order-reduced HFGMC for linearly elastic constituents strongly depends on the number of subcells in the RUC. In this study, four distinct RUCs with a nominal $60 \%$ fiber volume fraction and a random microstructure were generated using a recently developed RUC generator [34]. These RUCs have 256, 1024, 2116, and 5184 subcells and are shown in Figures 2(a)-2(d), respectively. Furthermore, the number of subcells occupied by elastic materials was fixed at $60 \%$ in order to more accurately compare results for the different size RUCs.
In the HFGMC, a combination of global stress or strain components and a temperature change can be applied to an RUC. For this study, a $2 \%$ normal strain in the $y_{2}$-direction (cf. Figure 1(b)) was applied over 150 loading increments to each of the four RUCs in Figure 2. With the exception of the axial stress in the $y_{2}$-direction, all other applied stress components were set to zero. Additionally, a linear temperature increase from $-25^{\circ} \mathrm{C}$ to $50^{\circ} \mathrm{C}$ was applied. The temperature and mechanical loads were imposed simultaneously in order to require assembly and solution of the HFGMC equations for each loading increment which represents the most general 
loading case. A total of 49 integration points were assigned to each subcell. This number was found to be the minimum necessary to achieve convergence of the inelastic strain/stress field for the reference solution. A fixed, conservative number of iterations (i.e., 50) were performed for each increment. Since multiple unique RUCs were considered in this study, no robust criterion was specified to establish convergence of the inelastic fields. Rather, analyses were performed to establish an appropriate fixed number of iterations necessary for convergence for all RUCs. By basing convergence on a fixed number of iterations, an appropriate comparison of the computational cost for the different RUCs could be performed without having to consider whether fewer/more iterations were required for a particular RUC analysis.

4.2. Generation of the Order-Reduced HFGMC Models. As previously mentioned, the method of snapshots was used to determine the orthonormal basis vectors required to approximate the reference solution and project to the reduced subspace. This technique requires that the solution to each of HFGMC system of equations be obtained at a number of time/loading intervals (snapshots). For instance, Ricks et al. [17] obtained snapshots by independently varying the six strain components, and an SVD was performed to determine the orthonormal basis vectors. These basis vectors are substituted into (7) and used to generate an order-reduced model. This process was performed offline prior to performing an analysis of interest. For linearly elastic materials without any temperature change, the effective elastic stiffness tensor for the RUC was calculated when generating the snapshots. This tensor can be stored to eliminate the need to calculate the mechanical strain concentration tensor in the order-reduced HFGMC. As such, Ricks et al. [17] demonstrated that a single order-reduced model can accurately and efficiently represent the full HFGMC system of equations. Additionally, for isothermal elasticity, a solution to the HFGMC system of equations is only required once per increment (no need for an iterative procedure).

However, when thermoinelastic materials are considered, eight unique HFGMC systems of equations must be repeatedly assembled and solved in order to determine the mechanical strain concentration tensor and the solution due to the applied loading conditions. More details concerning these eight systems of equations can be found in the appendix. A single order-reduced model is unlikely to accurately and efficiently represent all eight systems of equations. For simplicity, in this work, a family of eight order-reduced models were used in an analysis of each RUC. The first six of the eight order-reduced models were used to establish the mechanical strain concentration tensor. The remaining two models were used to solve the actual problem with the applied loading conditions.

In order to establish the eight order-reduced models, the previously described loading conditions (Section 4.1) were applied to each RUC. The converged solution at each of 150 temperature increments for each set of equations was used to populate eight snapshot matrices (one for each unique set of equations). An SVD was performed on each snapshot matrix and was used to generate a set of orthonormal basis

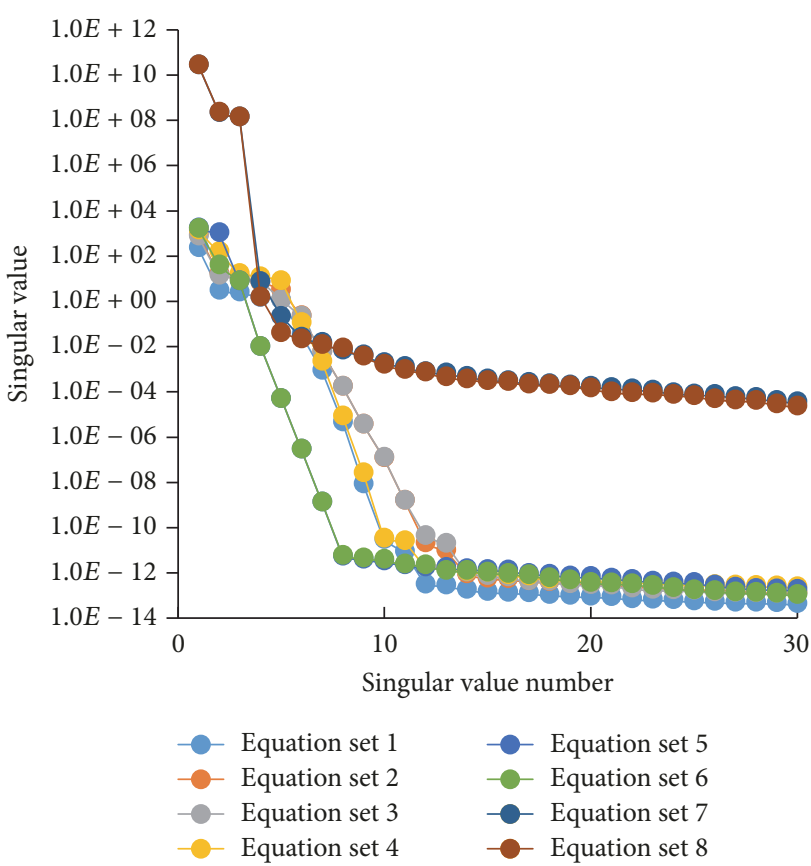

FIGURE 3: Singular values of the snapshot matrix for each of eight sets of simultaneous equations for a 256 subcell RUC.

vectors. Figure 3 contains a plot of the first 30 singular values associated with each of the eight order-reduced models for the 256 subcell RUC (i.e., Figure 2(a)). The singular values for each of the eight solutions decreased by several orders of magnitude over the first ten singular values. Similar plots were obtained for the other 1024, 2116, and 5184 subcell RUCs. This suggested that accurate order-reduced models could likely be generated using a relatively small number of basis vectors. Conceivably, each of the eight order-reduced HFGMC models for a given RUC can require a distinct number of basis vectors. Since the plot of the singular values was similar for the first six models (used to establish effective properties) and the boundary conditions are similar, a constant number of basis vectors was used for the first six models for each RUC configuration. Similarly, a different number of basis vectors was used for the remaining two models (used to solve the actual boundary value problem). Future studies will investigate more robust methods to establish the appropriate size of each order-reduced model within HFGMC.

4.3. Assessment of the Order-Reduced HFGMC Models for Determining Effective Properties. The accuracy of the family of order-reduced models for each of the 256, 1024, 2116, and 5184 subcell RUCs was assessed by performing multiple simulations, each with a different number of basis vectors. Recall that the first six of eight order-reduced models for a given RUC are used to establish RUC effective properties. Since these properties influence the global fields, the error in the effective elastic stiffness and thermal stress tensors was determined by performing a series of analyses using one to ten basis vectors $(k=1,2, \ldots, 10)$. Recall that, for this study, the effective properties do not depend on the 


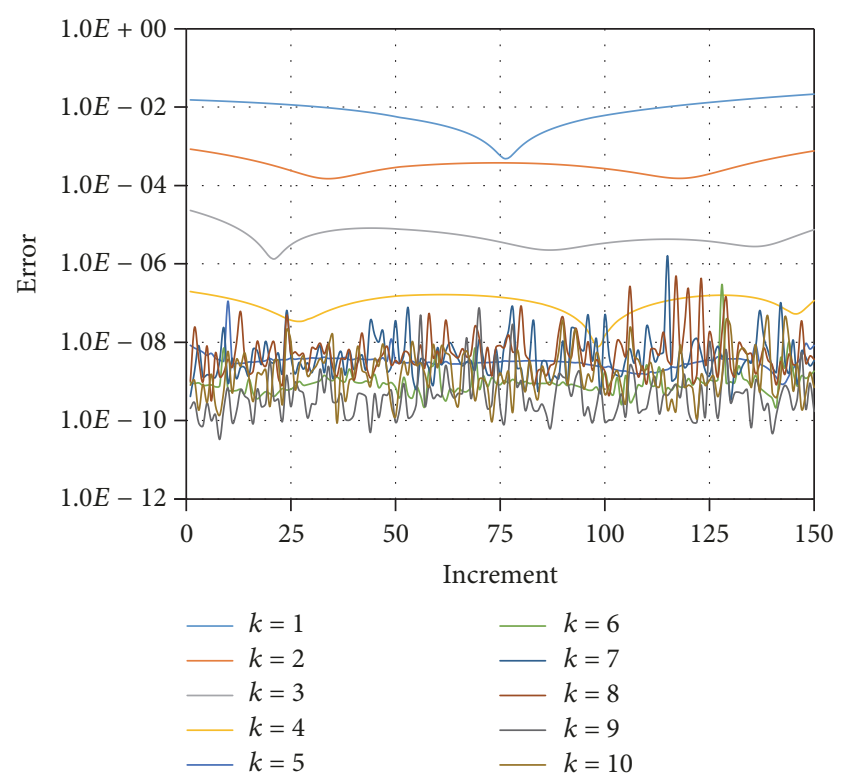

FIgURE 4: Error in the effective elastic stiffness tensor for the 256 subcell RUC of an E-glass/Nylon 12 composite at each temperature increment for the first six of eight order-reduced models each containing $k=1-10$ basis vectors.

inelastic state or applied mechanical loading and are only a function of temperature. Hence, the temperature was varied over 150 increments from $-25^{\circ} \mathrm{C}$ to $50^{\circ} \mathrm{C}$ and only the effective properties were determined at each temperature. The error associated with each temperature increment was calculated using the following relationship:

$$
\operatorname{Err}=\frac{\left\|\mathbf{A}_{\mathbf{R}}-\mathbf{A}_{\mathrm{OR}}\right\|_{2}}{\left\|\mathbf{A}_{\mathbf{R}}\right\|_{2}},
$$

where A represents either the vectorized effective elastic stiffness tensor or the effective thermal stress tensor, the subscripts $\mathbf{R}$ and $\mathbf{O R}$ correspond to the reference or orderreduced vectors, respectively, and $\|\cdot\|_{2}$ denotes the $L_{2^{-}}$ norm. Figure 4 shows the error in the effective elastic stiffness tensor for the 256 subcell RUC at each temperature increment for order-reduced models containing one to ten basis vectors. The error was observed to be relatively constant as the temperature varied for a given model. Furthermore, as the number of basis vectors used in the order-reduced models increased $(k \geq 5)$, the error became increasingly negligible. Similar trends were observed for the error in the effective thermal stress tensor. Since both the effective elastic stiffness and thermal stress tensors are calculated using the mechanical strain concentration tensor [1], the two tensors have similar errors for order-reduced models with the same number of basis vectors. Analogous error estimates in the effective properties for the 1024, 2116, and 5184 subcell RUCs were obtained and closely resembled those of the 256 subcell RUC. Hence, for all RUCs, $k=5$ was determined to yield accurate effective properties and was used in subsequent assessments of the subcell/global fields. In essence, the first six of eight order-reduced models for a given RUC involved

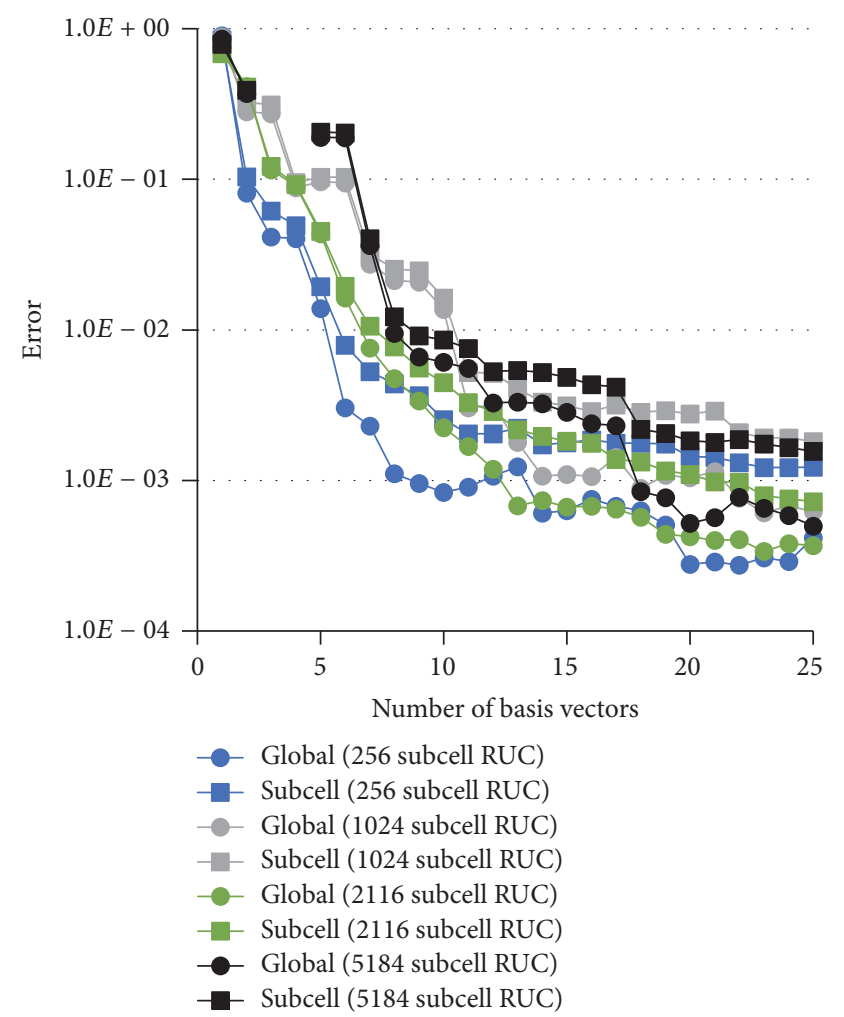

FigURE 5: Average error in the global/subcell stresses for each RUC of an E-glass/Nylon 12 composite for order-reduced models (last two of eight) with varying numbers of basis vectors.

solving a dense set of $k=5$ equations, while the reference (unreduced) model was comprised of a highly sparse set of $n=1536-31,104$ equations depending on the RUC architecture considered.

4.4. Assessment of the Order-Reduced HFGMC Models for Determining Global/Local Fields. In order to assess the accuracy of the remaining two order-reduced models for each RUC architecture, the global and subcell stress fields were evaluated where the number of basis vectors was varied, $k$ $=1-25$, for each model. Recall that these two order-reduced models were used to determine global/local fields under the applied loading conditions. As previously mentioned, each of these two models used the same number of basis vectors for a given simulation. The error in the global/subcell stress field was calculated using (8), where $\mathbf{A}$ corresponded to the $6 \times 1$ global or subcell stress vector. The error in subcell stresses was averaged across all iterations for each RUC for a given number of basis vectors. Similarly, for each RUC, the error in subcell stresses was determined for each subcell at all iterations and then averaged. Figure 5 contains a plot of the average error in global/subcell stresses as a function of the number of basis vectors $(k)$ for each of the $256,1024,2116$, and 5184 subcell RUCs. In general, as the number of basis vectors in the last two order-reduced models increased, the average error in both the global and subcell stresses decreased by orders of magnitude. Not surprisingly, the average error in subcell stresses was typically greater 


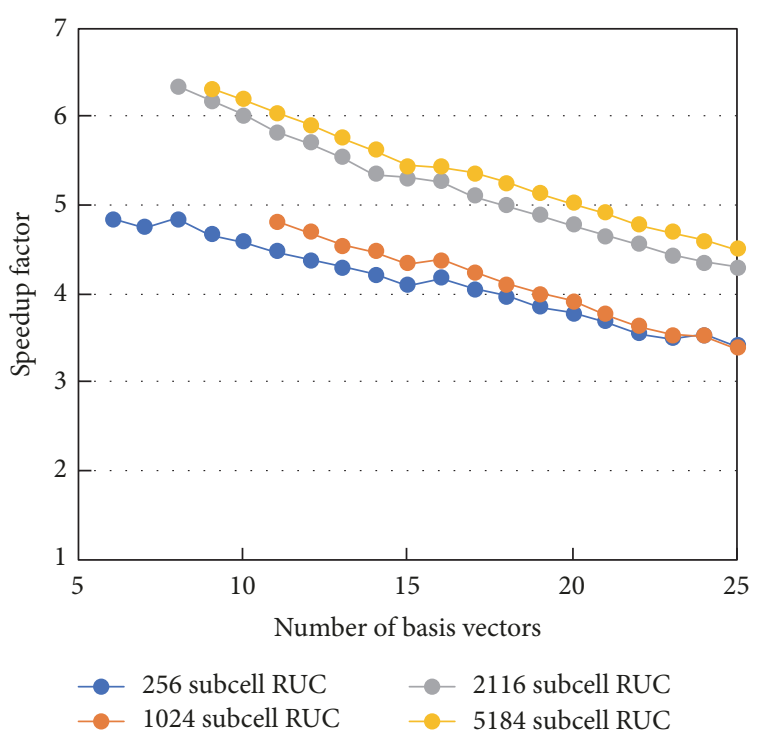

(a)

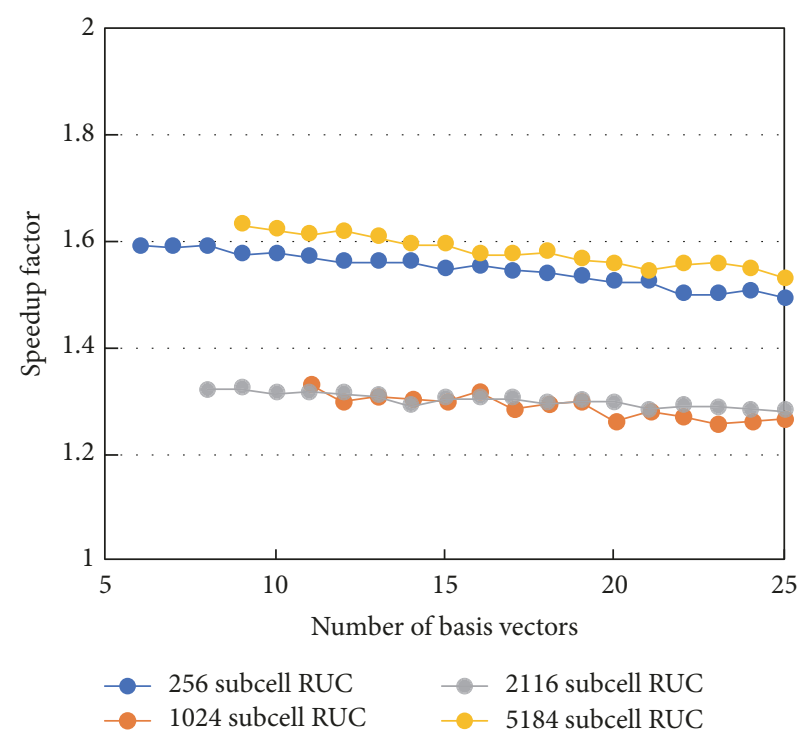

(b)

FIGURE 6: Speedup factors calculated from the (a) assembly/solution step runtimes and (b) total runtimes for each RUC as a function of the number of basis vectors used in the last two of eight order-reduced models.

than that of the global (continuum-averaged) stresses. Minor differences in the order-reduced approximations will lead to larger fluctuations in the calculated local subcell stress fields than for the global (homogenized) stress field. Hence, more basis vectors will be required to obtain a desired level of accuracy in the calculated subcell fields than that required to obtain the same accuracy in the global fields. Of course, such results are also highly dependent on the local distribution of features and properties within a given RUC, as well as the applied thermomechanical loading. For example, an order-reduced model of the 1024 subcell RUC (Figure 2(b)) with 11 basis vectors is required to calculate global/subcell stresses with an error of less than $1 \%$ relative to the reference (unreduced) model (Figure 5). In contrast, only nine basis vectors are required to achieve a similar level of accuracy for the more complex and highly discretized 5184 subcell RUC (Figure 2(d)). Similarly, for a given number of basis vectors, the estimated error for an order-reduced model of the 1024 subcell RUC (Figure 2(b)) exceeds that for the more highly refined 2116 subcell RUC containing significantly more E-glass fibers (Figure 2(c)). Hence, the optimal size of the order-reduced model necessary to simulate a given RUC is problem specific and driven by local features (i.e., material distribution or regions of intense inelasticity), as well as the applied loading. Nonetheless, the error associated with each of the order-reduced models of the 256, 1024, 2116, and 5184 subcell RUCs became exceedingly small once the number of basis vectors exceeded $k \geq 10-15$. Additionally, some local instabilities were observed for the 5184 subcell RUC for smaller order-reduced models $(k=3,4)$. These instabilities are likely the result of the order-reduced model not being accurate for such a low number of basis vectors.
The computational efficiency of the order-reduced models was assessed for each RUC architecture. The time spent assembling/solving the HFGMC systems of equations was determined for the reference model for each RUC and the corresponding family of order-reduced models. Since each order-reduced model requires mapping to and from the order-reduced system of equations at each increment/ iteration (i.e., additional matrix multiplications are required), the computation runtimes for the order-reduced models include both the mapping operations and iterative solution of eight sets of simultaneous equations. The total runtime for a given RUC analysis was also determined. The total runtime had two main contributions: (i) the eight separate equation assembly/solution steps and (ii) the localization step used to calculate the inelastic field at individual integration points. These two factors represented $>90 \%$ of the total analysis runtime. Note that read/write operations involving storage of local subcell field quantities were not included in this performance assessment since such operations are unaffected by the order-reduction procedures.

Speedup factors were calculated by dividing the appropriate reference solution runtime by the order-reduced runtime, where the minimum number of basis vectors was selected such that the error in the average subcell stresses did not exceed $1 \%$. As a reminder, $k=5$ basis vectors were employed in the first six sets of eight order-reduced HFGMC equations. Figure 6 shows the effect of varying the number of basis vectors in the latter two sets of order-reduced equations on speedup factors. The speedup factors for (i) the equation assembly/solution and (ii) total analysis runtimes are shown in Figures 6(a) and 6(b), respectively, for the order-reduced models for each of the RUCs. Regardless of the RUC architecture, as the number of basis vectors used in the last two 
order-reduced models increased, the speedup factor from the equation assembly/solution steps decreased proportionally. More importantly, for a given number of basis vectors, the more highly discretized and complex order-reduced 2116 and 5184 subcell RUC models displayed markedly higher assembly/solution speedup factors than did the less refined 256 and 1024 subcell models. This trend is consistent with previously reported results for RUCs with elastic constituents [17]. Regardless of the number of basis vectors employed and the RUC architecture, significant computational savings in the equation assembly/solution steps were achieved. For instance, order-reduced models with less than $1 \%$ error in the subcell stress fields were 4.8-6.3 times faster than the reference solution (Figure 6(a)). This underscores the advantage of using order-reduction techniques in the multiscale analysis of materials with complex microstructures.

Interestingly, the significant improvement in equation assembly/solution step runtimes associated with each of the order-reduced RUC models did not translate into a similar improvement in the total runtime. The speedup factors in total runtimes varied, 1.3-1.6, depending on the RUC architecture and were relatively insensitive to the number of basis vectors (Figure 6(b)). In spite of substantial improvements in equation assembly/solution step runtimes associated with the order-reduced models, this difference between the total runtimes for the order-reduced and reference models appears dominated by the fraction of the total runtime spent numerically evaluating the inelastic field quantities at each integration point. The procedure used to assess the Nylon 12 matrix inelasticity was identical for the reference model and order-reduced models. Depending on the RUC architecture, roughly $50-70 \%$ of the total runtime was dedicated to evaluation of the inelastic field. Hence, the benefits of order-reduction can only be realized for the remaining $26-46 \%$ of the total runtimes associated with equation assembly/solution processes. Nonetheless, use of order-reduction techniques resulted in a $21-38 \%$ reduction in total runtimes. The improvement in computational efficiency may become more pronounced for RUCs containing fewer inelastic matrix subcells. As an aside, the fraction of the total runtime spent assembling/solving the HFGMC equations seems to be more dependent on the local distribution of properties within an RUC rather than the level of refinement and/or complexity of a given RUC. Additional analyses for increasingly refined ordered microstructures (rather than the random ones considered in this study) yielded similar results.

The order-reduction procedures presented herein resulted in up to a sixfold increase in the computational efficiency associated with equation assembly/solution processes in HFGMC. The relative improvement in computational runtimes was not as pronounced as for traditional FE orderreduced approaches that involve an iterative solution of simultaneous equations with a sparse, symmetric, and banded stiffness matrix. In contrast, the nonlinear HFGMC simultaneous equations contain a sparse, unsymmetrical, unbanded, and ill-conditioned stiffness matrix. Moreover, significantly more integration points are required to achieve a converged inelastic field in HFGMC. Despite these disadvantages, the nonlinear HFGMC is intrinsically faster than traditional (unreduced) FE analyses [1]. The relative improvement in computational runtimes for order-reduced HFGMC models, however, will likely be lower than for order-reduced FE models. In the future, the efficiency of order-reduced HFGMC models can be improved by implementing direct assembly techniques that can be used to apply the order-reduction at the RUC subcell equations rather than the fully assembled global equations $[35,36]$. Additionally, although this study considered an Eglass/Nylon 12 material system, similar computational results will likely be obtained for these RUC architectures if different constituents are used. Minor differences may arise due to the numerical solution of the individual systems of equations. By coupling improved plasticity algorithms with direct assembly techniques, the order-reduction HFGMC model developed in this work can provide significant computational savings over the traditional procedure. This improvement is essential for developing higher-fidelity multiscale analysis procedures.

\section{Conclusions}

In this study, the High-Fidelity Generalized Method of Cells (HFGMC) global system of $n \times n$ equations for doubly periodic repeating unit cells (RUCs) comprised of E-glass fibers and a Nylon 12 matrix was reduced in size through the use of Proper Orthogonal Decomposition with Petrov-Galerkin projection. The order-reduced system of equations was compared to the unmodified HFGMC equations for micromechanics models with four distinct RUCs of increasing complexity (256 to 5184 subcells). For all RUCs, relatively small order-reduced models were found to accurately reproduce effective properties (five basis vectors) and global/subcell stresses (six to eleven basis vectors). A 4.8-6.3x speedup in the equation assembly/solution process was achieved while not exceeding a $1 \%$ error in the average subcell stresses. Moreover, total runtimes were reduced by 21-38\%. The discrepancy between the speedup of the assembly/solution procedure and the total runtime is attributable to the fact that a significant fraction of the computational time in HFGMC is spent evaluating inelastic fields. Such calculations do not benefit from the use of order-reduction procedures. Current work is aimed at further improving the computational efficiency of the order-reduced HFGMC by performing order-reduction at the subcell level rather than the global level.

\section{Appendix}

\section{Brief Overview of the Eight Sets of HFGMC Equations}

In a typical nonlinear HFGMC analysis, eight unique sets of equations are generated and solved. This section contains a brief overview describing the nature of these equations. The reader is referred to $[1,37]$ for more details regarding the specific numerical implementation. 
In the HFGMC, a second-order expansion of the subcell displacement field is performed and is given by

$$
\begin{aligned}
u_{i}{ }^{(\beta \gamma)}= & \bar{\varepsilon}_{i j} x_{j}+W_{i(00)}^{(\beta \gamma)}+\bar{y}_{2}{ }^{(\beta)} W_{i(10)}^{(\beta \gamma)}+\bar{y}_{3}{ }^{(\gamma)} W_{i(01)}^{(\beta \gamma)} \\
& +\frac{1}{2}\left(3 \bar{y}_{2}^{(\beta)^{2}}-\frac{h_{\beta}^{2}}{4}\right) W_{i(20)}^{(\beta \gamma)} \\
& +\frac{1}{2}\left(3 \bar{y}_{3}{ }^{(\gamma)^{2}}-\frac{l_{\gamma}^{2}}{4}\right) W_{i(02)}^{(\beta \gamma)} .
\end{aligned}
$$

For perfect elasticity, a linear variation in strain and stress within a subcell results from the displacement field given by (A.1). When thermoinelasticity is admitted, a higher-order representation of the subcell strain and stress field is required $[1,37]$. The subcell strain field can be expressed using a Legendre polynomial, $P$, expansion by

$$
\begin{aligned}
& \varepsilon_{i j}{ }^{(\beta \gamma)}=\sum_{m=0}^{\infty} \sum_{n=0}^{\infty} \sqrt{(1+2 m)(1+2 n)} e_{i j(m n)}^{(\beta \gamma)} P_{m}\left(\zeta_{2}{ }^{(\beta)}\right) \\
& \cdot P_{n}\left(\zeta_{3}{ }^{(\gamma)}\right)
\end{aligned}
$$

where nondimensional variables $\zeta_{i}^{(\cdot)}$ map the subcell coordinates onto the interval $-1 \leq \zeta_{i}^{(\cdot)} \leq 1$ (i.e., $\zeta_{2}^{(\beta)}=2 \bar{y}_{2}{ }^{(\beta)} / h_{\beta}$ and $\left.\zeta_{3}{ }^{(\gamma)}=2 \bar{y}_{3}{ }^{(\gamma)} / l_{\gamma}\right)$ and $e_{i j(m n)}^{(\beta \gamma)}$ are the strain coefficients. The average strains in a subcell are given by $e_{i j(00)}^{(\beta \gamma)}[1,37]$. A similar expression to (A.2) for the subcell stresses can also be derived. The average total subcell strains can then be related to the RUC-averaged applied total strains, $\overline{\boldsymbol{\varepsilon}}$, through

$$
\mathbf{e}_{(00)}^{(\beta \gamma)}=\mathbf{A}^{(\beta \gamma)} \overline{\boldsymbol{\varepsilon}}+\mathbf{D}^{(\beta \gamma)}
$$

where $\mathbf{A}^{(\beta \gamma)}$ is the $6 \times 6$ mechanical strain concentration matrix for the subcell and $\mathbf{D}^{(\beta \gamma)}$ is a $6 \times 1$ vector that accounts for current thermoinelastic effects in the subcell. $\mathbf{A}^{(\beta \gamma)}$ is used to calculate RUC-averaged effective properties and is found by independently setting the components of $\overline{\boldsymbol{\varepsilon}}$ to unity. At the same time, all other RUC-averaged total strain components and thermoinelastic effects (i.e., $\mathbf{D}^{(\beta \gamma)}$ ) are set to zero. This procedure is similar to finite-element based homogenization techniques [38]. For example, the first column of $\mathbf{A}^{(\beta \gamma)}$ can be found by setting $\bar{\varepsilon}_{11}=1$ and all other strain components equal to zero along with $\mathbf{D}^{(\beta \gamma)}=0$. Practically, this is implemented by assembling and solving a set of equations given by (3). In this case, $\mathbf{f}=\mathbf{f}(\overline{\boldsymbol{\varepsilon}}), \mathbf{g}=0$ (no thermoinelastic effects), and the solution, $\mathbf{U}$, contains the surface-averaged fluctuating displacements in each subcell, $\overline{\mathbf{u}}^{(\beta \gamma)}$. The relationships between $\overline{\mathbf{u}}^{(\beta \gamma)}$ and $\mathbf{e}_{(00)}^{(\beta \gamma)}$ can be found in $[1,37]$. Using (A.3), the first column of $\mathbf{A}^{(\beta \gamma)}$ is then equal to $\mathbf{e}_{(00)}^{(\beta \gamma)}$. Six unique sets of equations are therefore required in order to calculate $\mathbf{A}^{(\beta \gamma)}$. The relationships between $\mathbf{A}^{(\beta \gamma)}$ and the RUC-averaged effective properties can be found in $[1,37]$. This step must be performed at each loading increment during which the subcell material properties change (e.g., due to temperature or damage). Furthermore, as previously mentioned, the stiffness matrix, $\mathbf{K}$, for each of these equations is identical.

The remaining two sets of equations are assembled and solved iteratively at each loading increment and are used to determine the subcell elastic and inelastic fields. For both of these sets, $\mathbf{K}$ remains unchanged and is independent of any inelastic effects. A seventh set of equations (same form as (3)) based on the current applied loading conditions and inelastic state can be assembled, solved, and used to calculate the subcell total strains and stresses. Similar to the procedure for calculating effective properties, the eighth set of equations is used to calculate the thermoinelastic vector, $\mathbf{D}^{(\beta \gamma)}$, by setting $\overline{\boldsymbol{\varepsilon}}=0$. The relationships between $\mathbf{D}^{(\beta \gamma)}$ and RUC-averaged inelastic strains/stresses can be found in $[1,37]$. The RUCaveraged inelastic strains are then used to modify $\overline{\boldsymbol{\varepsilon}}$ at the next iteration. This iterative procedure is described in more detail in [37]. At the end of an iteration, convergence can be verified by checking against some user-specified criterion. Alternatively, a sufficiently high number of iterations can be performed to ensure that the local inelastic fields have converged.

\section{Conflicts of Interest}

The authors declare that there are no conflicts of interest regarding the publication of this paper.

\section{Acknowledgments}

This work was supported by the National Aeronautics and Space Administration's Composite Technology for Exploration project.

\section{References}

[1] J. Aboudi, S. M. Arnold, and B. A. Bednarcyk, Micromechanics of composite materials: A generalized multiscale analysis approach, Butterworth-Heinemann, Oxford, UK, 2012.

[2] Y. C. Liang, H. P. Lee, S. P. Lim, W. Z. Lin, K. H. Lee, and C. $\mathrm{G}$. Wu, "Proper orthogonal decomposition and its applications. I. Theory," Journal of Sound and Vibration, vol. 252, no. 3, pp. 527-544, 2002.

[3] A. Chatterjee, "An introduction to the proper orthogonal decomposition," Current Science, vol. 78, no. 7, pp. 808-817, 2000.

[4] F. Chinesta, P. Ladeveze, and E. Cueto, "A Short Review on Model Order Reduction Based on Proper Generalized Decomposition," Archives of Computational Methods in Engineering: State-of-the-Art Reviews, vol. 18, no. 4, pp. 395-404, 2011.

[5] F. Chinesta, A. Ammar, and E. a. Cueto, "Recent advances and new challenges in the use of the proper generalized decomposition for solving multidimensional models," Archives of Computational Methods in Engineerin: State-of-the-Art Reviews, vol. 17, no. 4, pp. 327-350, 2010.

[6] K. Carlberg, C. Bou-Mosleh, and C. Farhat, "Efficient nonlinear model reduction via a least-squares Petrov-Galerkin projection and compressive tensor approximations," International Journal for Numerical Methods in Engineering, vol. 86, no. 2, pp. 155-181, 2011. 
[7] G. Berkooz, P. Holmes, and J. L. Lumley, “The proper orthogonal decomposition in the analysis of turbulent flows," Annual Review of Fluid Mechanics, vol. 25, no. 1, pp. 539-575, 1993.

[8] K. Kunisch and S. Volkwein, "Galerkin proper orthogonal decomposition methods for a general equation in fluid dynamics," SIAM Journal on Numerical Analysis, vol. 40, no. 2, pp. 492515, 2002.

[9] S. S. Ravindran, "A reduced-order approach for optimal control of fluids using proper orthogonal decomposition," International Journal for Numerical Methods in Fluids, vol. 34, no. 5, pp. 425448, 2000.

[10] A. Radermacher, B. A. Bednarcyk, B. Stier, J. Simon, L. Zhou, and S. Reese, "Displacement-based multiscale modeling of fiber-reinforced composites by means of proper orthogonal decomposition," Advanced Modeling and Simulation in Engineering Sciences, vol. 3, no. 1, 2016.

[11] A. Radermacher, S. Reese, and A. M. Hadoush, "Selective proper orthogonal decomposition model reduction for forming simulations," PAMM, vol. 13, no. 1, pp. 115-116, 2013.

[12] J. A. Hernández, J. Oliver, A. E. Huespe, M. A. Caicedo, and J. C. Cante, "High-performance model reduction techniques in computational multiscale homogenization," Computer Methods Applied Mechanics and Engineering, vol. 276, pp. 149-189, 2014.

[13] J. Yvonnet and Q.-C. He, "The reduced model multiscale method (R3M) for the non-linear homogenization of hyperelastic media at finite strains," Journal of Computational Physics, vol. 223, no. 1, pp. 341-368, 2007.

[14] P. Kerfriden, P. Gosselet, S. Adhikari, and S. P. A. Bordas, "Bridging proper orthogonal decomposition methods and augmented Newton-Krylov algorithms: an adaptive model order reduction for highly nonlinear mechanical problems," Computer Methods Applied Mechanics and Engineering, vol. 200, no. 5-8, pp. 850866, 2011.

[15] F. Feyel, "A multilevel finite element method (FE2) to describe the response of highly non-linear structures using generalized continua," Computer Methods Applied Mechanics and Engineering, vol. 192, no. 28-30, pp. 3233-3244, 2003.

[16] F. Feyel and J.-L. Chaboche, "FE 2 multiscale approach for modelling the elastoviscoplastic behaviour of long fibre SiC/Ti composite materials," Computer Methods Applied Mechanics and Engineering, vol. 183, no. 3-4, pp. 309-330, 2000.

[17] T. M. Ricks, T. E. Lacy, E. J. Pineda, B. A. Bednarcyk, and S. M. Arnold, "Computationally efficient High-Fidelity Generalized Method of Cells micromechanics via order-reduction techniques," Composite Structures, vol. 156, pp. 2-9, 2016.

[18] D. Ryckelynck, "A priori hyperreduction method: An adaptive approach," Journal of Computational Physics, vol. 202, no. 1, pp. 346-366, 2005.

[19] S. Chaturantabut and D. C. Sorensen, "Nonlinear model reduction via discrete empirical interpolation," SIAM Journal on Scientific Computing, vol. 32, no. 5, pp. 2737-2764, 2010.

[20] R. Everson and L. Sirovich, "Karhunen-loève procedure for gappy data," Journal of the Optical Society of America A: Optics and Image Science, and Vision, vol. 12, no. 8, pp. 1657-1667, 1995.

[21] T. Bui-Thanh, M. Damodaran, and K. Willcox, "Aerodynamic data reconstruction and inverse design using proper orthogonal decomposition," AIAA Journal, vol. 42, no. 8, pp. 1505-1516, 2004.

[22] A. Bensoussan, J.-L. Lions, and G. Papanicolaou, Asymptotic Analysis for Periodic Structures, North-Holland, 1978.
[23] N. Bakhvalov and G. Panasenko, Homogenisation: averaging processes in periodic media, vol. 36 of Mathematics and its Applications (Soviet Series), Kluwer Academic Publishers Group, Dordrecht, 1989.

[24] E. Sanchez-Palencia and A. Zaoui, Homogenization techniques for composite media, vol. 272 of Lecture Notes in Physics, Springer-Verlag, Berlin, 1987.

[25] Y. Bansal and M.-J. Pindera, "Testing the predictive capability of the high-fidelity generalized method of cells using an efficient reformulation," Tech. Rep., Testing the predictive capability of the high-fidelity generalized method of cells using an efficient reformulation. Report Number, NASA/CR-2004, 2004.

[26] S. M. Arnold, B. A. Bednarcyk, and J. Aboudi, "Comparison of the computational efficiency of the original versus reformulated high-fidelity generalized method of cells," Report Number:NASA/TM-2004-213438, 2004.

[27] J. N. Reddy, An introduction to nonlinear finite element analysis - with applications to heat transfer, fluid mechanics, and solid mechanics, Oxford University Press, Oxford, Second edition, 2015.

[28] A. Mendelson, Plasticity: Theory and application, Macmillan, New York, 1968.

[29] B. A. Bednarcyk, J. Aboudi, and S. M. Arnold, "The equivalence of the radial return and Mendelson methods for integrating the classical plasticity equations," Computational Mechanics, vol. 41, no. 5, pp. 733-737, 2008.

[30] L. Sirovich, "Turbulence and the dynamics of coherent structures," Quarterly of Applied Mathematics, vol. 45, no. 3, pp. 561590, 1987.

[31] A. S. Kaddour, M. J. Hinton, P. A. Smith, and S. Li, "Mechanical properties and details of composite laminates for the test cases used in the third world-wide failure exercise," Journal of Composite Materials, vol. 47, no. 20-21, pp. 2427-2442, 2013.

[32] D. A. Şerban, G. Weber, L. Marşavina, V. V. Silberschmidt, and W. Hufenbach, "Tensile properties of semi-crystalline thermoplastic polymers: Effects of temperature and strain rates," Polymer Testing, vol. 32, no. 2, pp. 413-425, 2013.

[33] T. McNally, W. R. Murphy, C. Y. Lew, R. J. Turner, and G. P. Brennan, "Polyamide-12 layered silicate nanocomposites by melt blending," Polymer Journal, vol. 44, no. 9, pp. 2761-2772, 2003.

[34] P. Murthy and E. J. Pineda, "Tool for generation of MAC/GMC representative unit cell for CMC/PMC analysis," Report Number:NASA/TM-2016-219127, 2016.

[35] D. H. Allen and W. E. Haisler, Introduction to aerospace structural analysis, Wiley, New York, NY, USA, 1985.

[36] A. Radermacher, Proper orthogonal decomposition-based model reduction in nonlinear solid mechanics, Rheinisch-Westfälischen Technischen Hochschule Aachen University, 2014.

[37] J. Aboudi, M.-J. Pindera, and S. M. Arnold, "High-fidelity generalization method of cells for inelastic periodic multiphase materials," Report Number:NASA/TM-2002-211469, 2002.

[38] E. J. Barbero, Finite element analysis of composite materials using abaqus, CRC Press, Boca Raton, FL, USA, 2013. 


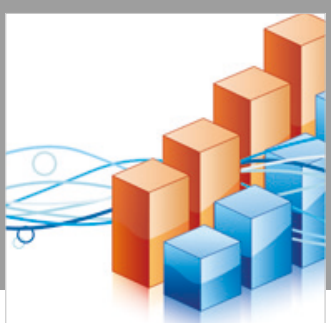

Advances in

Operations Research

\section{-n-m}
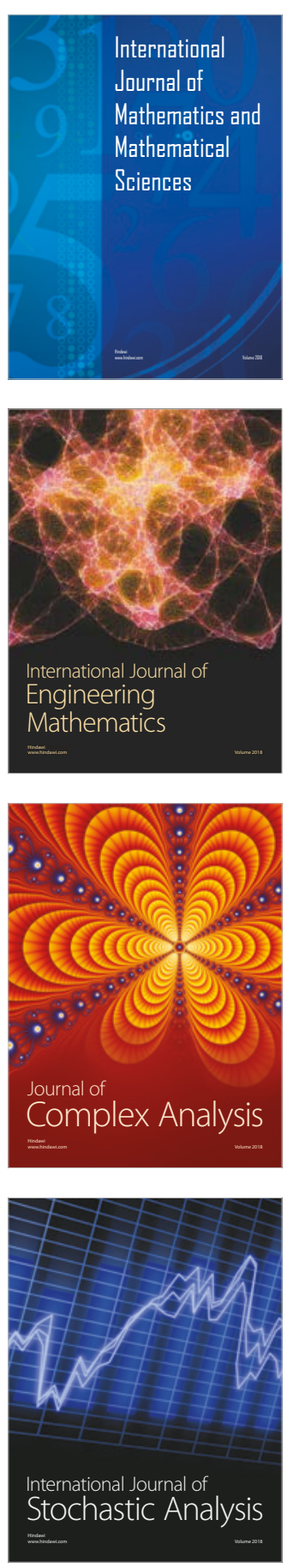
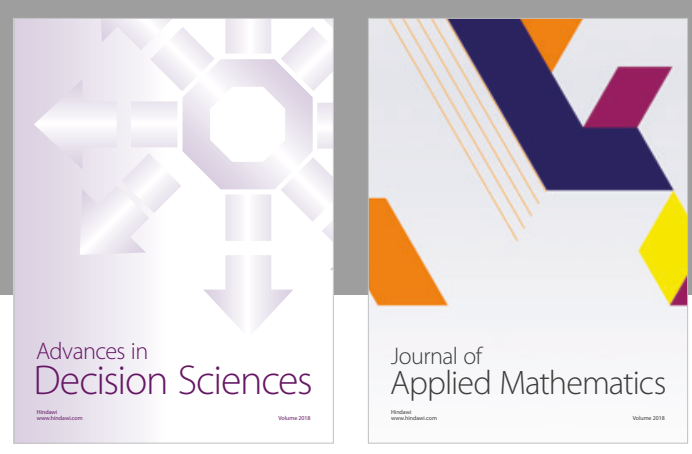

Journal of

Applied Mathematics
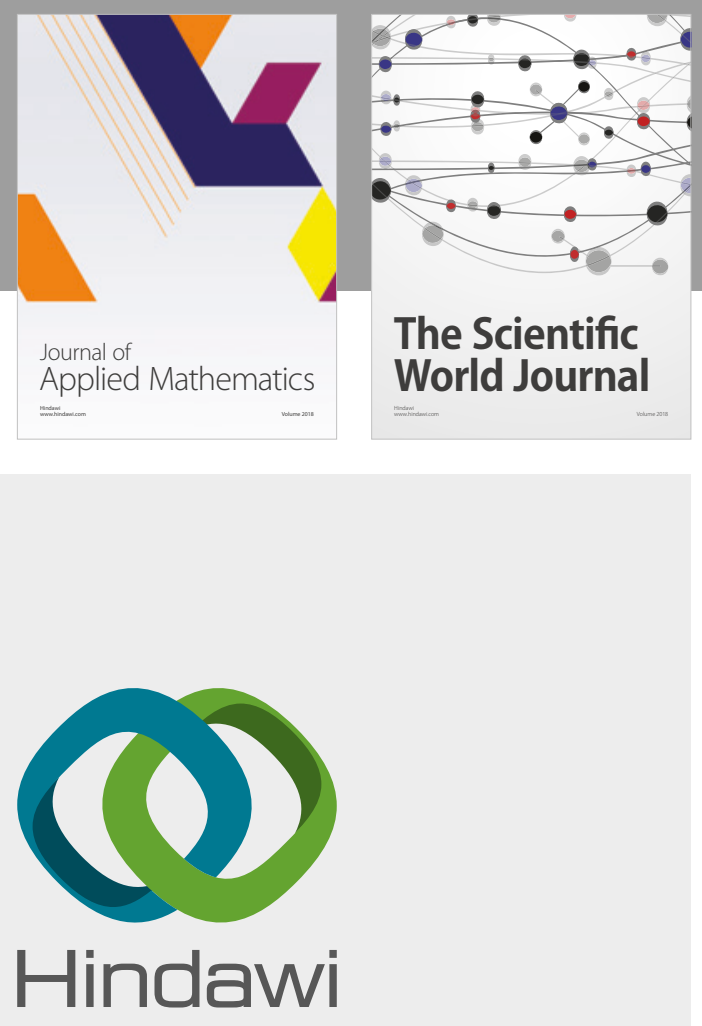

Submit your manuscripts at

www.hindawi.com

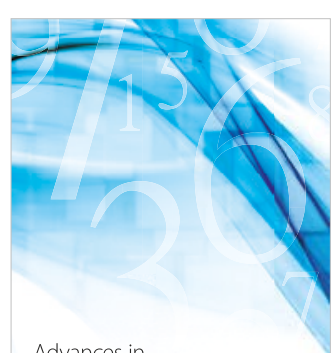

Advances in
Numerical Analysis
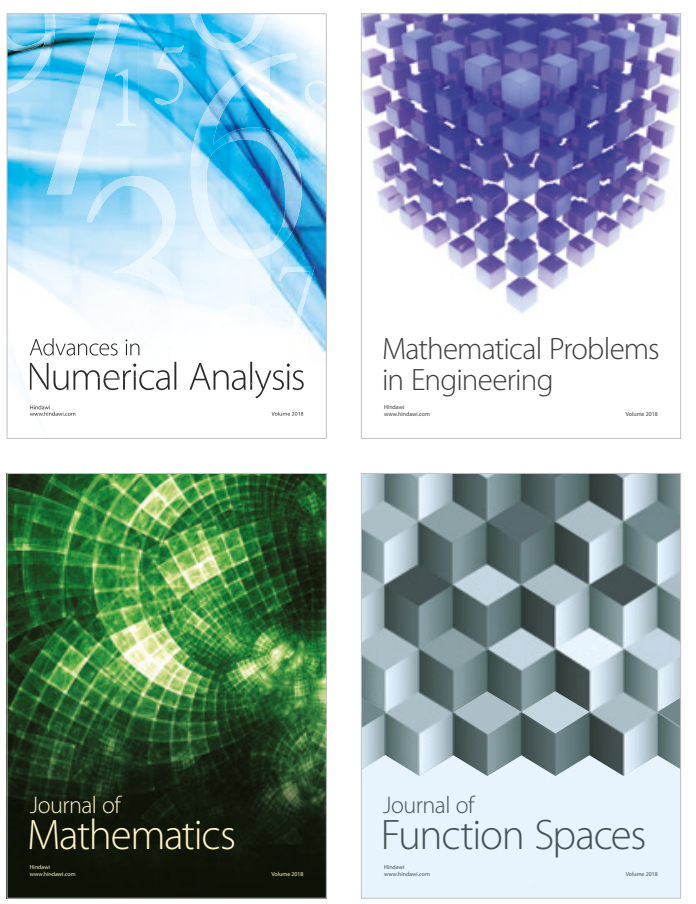

Mathematical Problems in Engineering

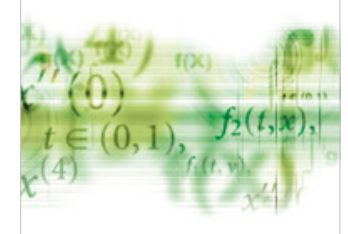

International Journal of

Differential Equations

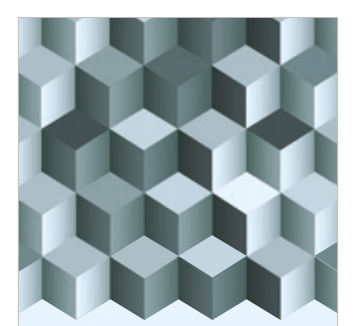

Journal of

Function Spaces

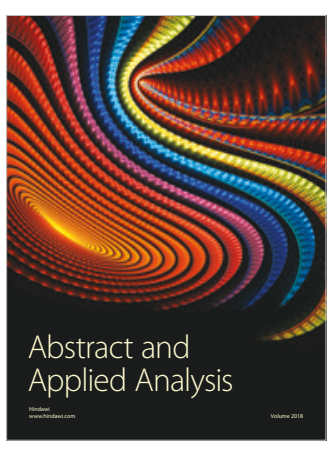

The Scientific

World Journal

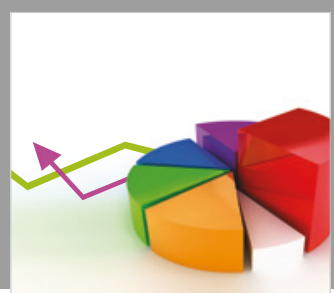

Journal of

Probability and Statistics
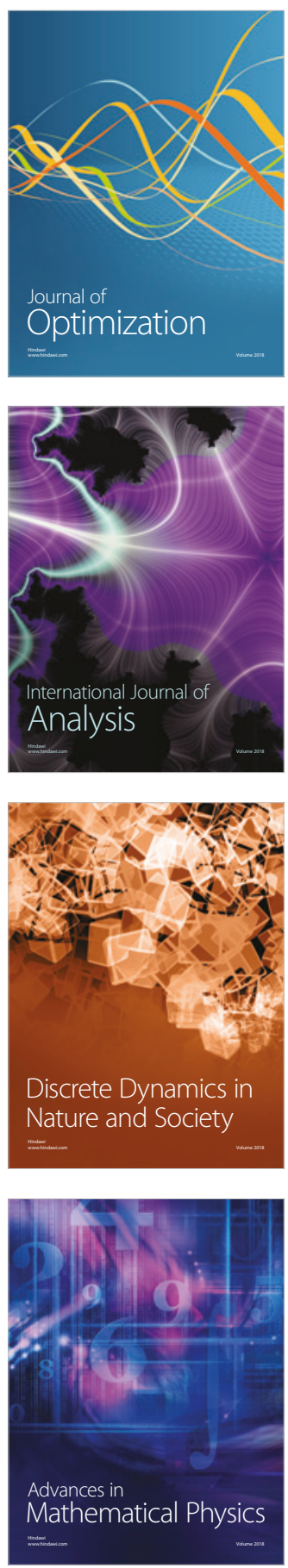